PROCEEDINGS OF THE

AMERICAN MATHEMATICAL SOCIETY

Volume 138, Number 9, September 2010, Pages 3163-3170

S 0002-9939(10)10214-7

Article electronically published on May 12, 2010

\title{
STABILITY OF WEIGHTED POINT EVALUATION FUNCTIONALS
}

\author{
JESÚS ARAUJO AND JUAN J. FONT
}

(Communicated by Nigel J. Kalton)

\begin{abstract}
Given $\epsilon>0$, a continuous linear functional $\varphi$ on $C(X)$ is said to be $\epsilon$-disjointness preserving if $|\varphi(f) \varphi(g)| \leq \epsilon$ whenever $f, g \in C(X)$ satisfy $\|f\|_{\infty}=\|g\|_{\infty}=1$ and $f g \equiv 0$. In this paper we provide the exact maximal distance from $\epsilon$-disjointness preserving linear functionals to the set of weighted point evaluation functionals.
\end{abstract}

\section{INTRODUCTION}

In 8, B.E. Johnson studied whether approximately multiplicative functionals on certain commutative Banach algebras can be approximated by multiplicative functionals. He showed that this question of stability for multiplicatively functionals is true on several classical algebras called AMNM algebras (almost multiplicative maps are near multiplicative maps). The algebra of continuous functions on a compact Hausdorff space or the group algebra of a locally compact abelian group belong to this class. Later, in [9, he pursued a similar study for approximately multiplicative maps defined between certain Banach algebras (AMNM pairs).

Recently, G. Dolinar (4]), in the spirit of the papers by B.E. Johnson cited above, considered a more general problem: the stability of disjointness preserving mappings defined between spaces of continuous functions. Let us first recall that a linear operator $T: C(X) \longrightarrow C(Y)$, with $X$ and $Y$ (nonempty) compact Hausdorff spaces, is said to be disjointness preserving (or separating) if, given $f, g \in C(X)$, $f g \equiv 0$ yields $(T f)(T g) \equiv 0$. Clearly every weighted composition map is disjointness preserving. Reciprocally, it is well known (see, for instance, [6, [1, [5], 7]) that if a disjointness preserving operator is continuous, then it is a weighted composition (where, as usual, the spaces of continuous functions are considered to be endowed with the sup norm $\|\cdot\|_{\infty}$ ).

On the other hand, given $\epsilon>0$, a continuous linear operator $T: C(X) \longrightarrow C(Y)$ is said to be approximately disjointness preserving or $\epsilon$-disjointness preserving ([4) if $\|(T f)(T g)\|_{\infty} \leq \epsilon$ whenever $f, g \in C(X)$ satisfy $\|f\|_{\infty}=\|g\|_{\infty}=1$ and $f g \equiv 0$ (or, equivalently, if $\|(T f)(T g)\|_{\infty} \leq \epsilon\|f\|_{\infty}\|g\|_{\infty}$ whenever $\left.f g \equiv 0\right)$.

Received by the editors June 15, 2009 and, in revised form, September 17, 2009.

2010 Mathematics Subject Classification. Primary 47B38; Secondary 46J10, 47B33.

Research of the first author was partially supported by the Spanish Ministry of Science and Education (Grant number MTM2006-14786).

Research of the second author was partially supported by the Spanish Ministry of Science and Education (Grant number MTM2008-04599) and by Bancaixa (Projecte P1-1B2008-26). 
One of the main results in [4 can be stated as follows: Let $\epsilon>0$ and let $T: C(X) \longrightarrow C(Y)$ be an $\epsilon$-disjointness preserving operator with $\|T\|=1$. Then there exists a weighted composition map $S: C(X) \longrightarrow C(Y)$ such that

$$
\|T-S\| \leq 20 \sqrt{\epsilon} \text {. }
$$

Recently, in [2, we have generalized completely Dolinar's result by proving that a bound for the stability of weighted composition operators is indeed $\sqrt{17 \epsilon / 2}$ rather than $20 \sqrt{\epsilon}$. An example is provided to show that $\sqrt{17 \epsilon / 2}$ is, in fact, a sharp bound.

In [3, among other things, we pursue our study of how far apart an $\epsilon$-disjointness preserving operator can be from the set of all weighted composition operators.

In both manuscripts ([2, [3]) we assume that $Y$ has at least two points, but in this paper we focus on continuous disjointness preserving linear functionals, that is, the case when $Y$ has just one point (so, if $C(X)^{\prime}$ denotes the space of linear and continuous functionals on $C(X), \varphi \in C(X)^{\prime}$ is said to be $\epsilon$-disjointness preserving if $|\varphi(f) \varphi(g)| \leq \epsilon$ whenever $f, g \in C(X)$ satisfy $\|f\|_{\infty}=\|g\|_{\infty}=1$ and $\left.f g \equiv 0\right)$. G. Dolinar also studied the stability of continuous disjointness preserving linear functionals on $C(X)$ and proved the following result (4. Theorem 1]): Let $\epsilon>0$ and let $\varphi \in C(X)^{\prime}$ be $\epsilon$-disjointness preserving with $\|\varphi\|=1$. Then there exists $\psi \in C(X)^{\prime}$ disjointness preserving such that

$$
\|\psi-\varphi\| \leq 3 \sqrt{\epsilon}
$$

Remark that, in this context, disjointness preserving linear functionals on $C(X)$ are precisely those of the form $\alpha \delta_{x}$, where $\alpha$ belongs to the scalar field $\mathbb{K}(=\mathbb{R}$ or $\mathbb{C})$ and $\delta_{x}$ is the evaluation functional at the point $x \in X$, that is, $\delta_{x}(f):=f(x)$ for every $f \in C(X)$.

In this paper we show that the situation in the context of continuous linear functionals is quite different from the general case treated in [2, 3], but, before stating our main result, we need some notation:

Throughout $X$ is assumed to have at least two points.

Given $\varphi \in C(X)^{\prime}$ and $r>0, B(\varphi, r)$ and $\bar{B}(\varphi, r)$ denote the open and the closed balls of center $\varphi$ and radius $r$, respectively. We will write $\lambda_{\varphi}$ to denote the measure which represents $\varphi$. For a regular measure $\lambda$, we will denote by $|\lambda|$ its total variation.

We denote by $\epsilon-\mathbf{D P}(X)$ the set of all norm one $\epsilon$-disjointness preserving functionals on $C(X)$ and by $\mathbf{W E}(X)$ the subset of $C(X)^{\prime}$ of elements of the form $\alpha \delta_{x}$, where $\alpha \in \mathbb{K}$ and $x \in X$.

For each $n \in \mathbb{N}$, we define

and

$$
\omega_{n}:=\frac{n^{2}-1}{4 n^{2}}
$$

$$
\mathbb{A}_{n}:=\left[\omega_{2 n-1}, \omega_{2 n+1}\right) .
$$

We now introduce the map $o_{X}:[0,1 / 4) \longrightarrow \mathbb{R}$, which depends on $\operatorname{card} X$ (the cardinality of $X$ ), as follows: For $n \in \mathbb{N}$ and $\epsilon \in \mathbb{A}_{n}$,

$$
o_{X}(\epsilon):=\left\{\begin{aligned}
\frac{2 n-1-\sqrt{1-4 \epsilon}}{2 n} & \text { if } 2 n \leq \operatorname{card} X, \\
\frac{2 m-1-\sqrt{1-4 \epsilon}}{2 m} & \text { if card } X=2 m<2 n, \\
\frac{2 m-2}{2 m-1} & \text { if card } X=2 m-1<2 n .
\end{aligned}\right.
$$

In this paper we prove, basically, that the maximal distance between elements $\varphi \in \epsilon-\mathbf{D P}(X)$ and the set $\mathbf{W E}(X)$ is $o_{X}(\epsilon)$, and that this distance is attained. 
Consequently, this result improves [4, Theorem 1] (cited above) by providing a sharp bound. We also study the behavior of this maximal distance for small and large $\epsilon$ (that is, those $\epsilon$ close to 0 and $1 / 4$, respectively).

\section{MAin RESUlts}

We begin this section by stating our main result.

Theorem 2.1. Let $0<\epsilon<1 / 4$. If $\varphi \in \epsilon-\mathbf{D P}(X)$, then

$$
\bar{B}\left(\varphi, o_{X}(\epsilon)\right) \cap \mathbf{W E}(X) \neq \emptyset \text {. }
$$

On the other hand, there exists $\varphi \in \epsilon-\mathbf{D P}(X)$ such that

$$
B\left(\varphi, o_{X}(\epsilon)\right) \cap \mathbf{W E}(X)=\emptyset .
$$

Remark 2.1. Sometimes the information given by the number $\epsilon$ is redundant in that $\epsilon$ is too "large" with respect to the cardinality of $X$. This happens, for instance, when $X$ is a set of $k$ points, where $k \in \mathbb{N}$ is odd. This is the reason why the definition of $o_{X}$ does not necessarily depend on $\epsilon$.

Remark 2.2. If $\varphi \in C(X)^{\prime}$ has norm 1, then it is $1 / 4$-disjointness preserving and, as a consequence, studying $\epsilon$-disjointness functionals is meaningless for $\epsilon \geq 1 / 4$. To see this, notice that if $f, g \in C(X)$ satisfy $\|f\|_{\infty}=\|g\|_{\infty}=1$ and $f g \equiv 0$, then $\|f+\alpha g\|_{\infty}=1$ for every $\alpha \in \mathbb{K}$ with $|\alpha|=1$, which implies that $|\varphi(f)+\alpha \varphi(g)| \leq 1$ and, in particular, $|\varphi(f)|+|\varphi(g)| \leq 1$. Hence, $|\varphi(f)||\varphi(g)| \leq 1 / 4$, and we are done.

The next result allows us to see which points in $(0,1)$ are those possible "maximal distances" we mentioned above. Obviously, the injectivity of $o_{X}$ indicates that each "maximal distance" corresponds to exactly one $\epsilon-\mathbf{D P}(X)$.

For $n \in \mathbb{N}$, we put

$$
\alpha_{n}:=\frac{n-1}{n}, \beta_{n}:=\frac{n^{2}-2}{n^{2}+n} .
$$

It is easy to see that $\alpha_{2 n-1}<\beta_{2 n}<\alpha_{2 n}$ for every $n \in \mathbb{N}$. We also define $V_{X}$ : $\bigcup_{n=1}^{\infty}\left[\alpha_{2 n-1}, \alpha_{2 n}\right) \longrightarrow[0,1 / 4)$ as

$$
V_{X}(\delta):=n(1-\delta)(1-n(1-\delta))
$$

for each $\delta \in\left[\alpha_{2 n-1}, \alpha_{2 n}\right)$.

Proposition 2.2. We have

im $o_{X}:=\left\{\begin{array}{cl}\bigcup_{n=1}^{\infty}\left[\alpha_{2 n-1}, \beta_{2 n}\right) & \text { if } X \text { is infinite, } \\ \left(\left[\alpha_{1}, \beta_{2}\right) \cup \cdots \cup\left[\alpha_{2 m-1}, \beta_{2 m}\right)\right) \cup\left[\beta_{2 m}, \alpha_{2 m}\right) & \text { if } \operatorname{card} X=2 m \in \mathbb{N}, \\ \left(\left[\alpha_{1}, \beta_{2}\right) \cup \cdots \cup\left[\alpha_{2 m-3}, \beta_{2 m-2}\right)\right) \cup\left\{\alpha_{2 m-1}\right\} & \text { if } \operatorname{card} X=2 m-1 \in \mathbb{N} .\end{array}\right.$

Also, if $X$ is infinite or card $X$ is even, then $o_{X}$ is injective, and if $\operatorname{card} X=$ $2 m-1$, then $o_{X}$ is injective exactly in $\left[0, \omega_{2 m-1}\right.$ ). The inverse of $o_{X}$ (where it exists) is given by the restriction of $V_{X}$ to im $o_{X}$ when $X$ is infinite or $\operatorname{card} X$ is even, and to im $o_{X} \backslash\left\{\alpha_{2 m-1}\right\}$ when $\operatorname{card} X=2 m-1$.

In particular, the above comments apply for every $X$ (with at least two points, as assumed) when $\epsilon$ is small enough, namely when $\epsilon \in \mathbb{A}_{1}$. In this case the restriction of the map $V_{X}$ defined above takes the form $V_{X}:(0,1 / 3) \longrightarrow(0,2 / 9), \delta \mapsto V_{X}(\delta):=$ $\delta(1-\delta)$. Notice that, in fact, $V_{X}(\delta)$ is the variance of a Bernoulli random variable of mean $\delta$. 
The following results are now immediate.

Corollary 2.3. Let

$$
\mathbf{A}:=\{\varphi: \exists \epsilon<1 / 4 \text { such that } \varphi \in \epsilon-\mathbf{D P}(X)\}
$$

and

$$
M:=\sup \{\operatorname{dist}(\varphi, \mathbf{W E}(X)): \varphi \in \mathbf{A}\} .
$$

Then $M=1$ if $X$ is infinite and $M=(k-1) / k$ if $k:=\operatorname{card} X$ is finite. Also the supremum is attained if and only if $X$ is a finite set with card $X$ odd.

Corollary 2.4. We have

$$
\lim _{\epsilon \rightarrow 0^{+}} \frac{o_{X}(\epsilon)}{\epsilon}=1
$$

and

$$
\lim _{\epsilon \rightarrow \frac{1}{4}^{-}} \frac{o_{X}(\epsilon)}{\epsilon}=\left\{\begin{aligned}
4 & \text { if } X \text { is infinite } \\
\frac{4(k-1)}{k} \quad & \text { if } k:=\operatorname{card} X \text { is finite. }
\end{aligned}\right.
$$

\section{Some Other RESUlts AND PROOFs}

Suppose that $X$ is a finite set of $k$ elements and that $\varphi \in C(X)^{\prime}$ has norm 1 . Then it is immediate that there exists a point $x \in X$ with $\left|\lambda_{\varphi}(\{x\})\right| \geq 1 / k$. We next see that this result can be sharpened when $k$ is even and $\varphi \in \epsilon-\mathbf{D P}(X)$, and also when $X$ has "many" elements (being finite or infinite).

For the sake of completeness, we first provide three technical lemmas whose proofs can be found in [2].

Lemma 3.1 ([2, Lemma 2.1]). Let $0<\epsilon<1 / 4$. Let $\varphi \in \epsilon-\mathbf{D P}(X)$ be positive. If $C$ is a Borel subset of $X$, then

$$
\lambda_{\varphi}(C) \notin\left(\frac{1-\sqrt{1-4 \epsilon}}{2}, \frac{1+\sqrt{1-4 \epsilon}}{2}\right) .
$$

If $\varphi \in C(X)^{\prime}$, then we put $|\varphi|(f):=\int_{X} f d\left|\lambda_{\varphi}\right|$ for every $f \in C(X)$.

Lemma 3.2 ([2, Lemma 2.2]). Given $\varphi \in C(X)^{\prime}$, then $|\varphi|$ is a positive linear functional on $C(X)$ with $\||\varphi|\|=\|\varphi\|$. Moreover, if $\epsilon>0$ and $\varphi \in \epsilon-\mathbf{D P}(X)$, then $|\varphi| \in \epsilon-\mathbf{D P}(X)$ and $\lambda_{|\varphi|}=\left|\lambda_{\varphi}\right|$.

Lemma 3.3 ([2, Lemma 2.3]). Let $0<\epsilon<1 / 4$. Let $\varphi \in \epsilon-\mathbf{D P}(X)$. Then there exists $x \in X$ with

$$
\left|\lambda_{\varphi}(\{x\})\right| \geq \sqrt{1-4 \epsilon} .
$$

Furthermore, if $0<\epsilon<2 / 9$, then there exists a unique $x \in X$ with

$$
\left|\lambda_{\varphi}(\{x\})\right| \geq \frac{1+\sqrt{1-4 \epsilon}}{2} .
$$

Proposition 3.4. Let $0<\epsilon<1 / 4$. Suppose that $X$ is a finite set of cardinality $k \in 2 \mathbb{N}$. If $\varphi \in \epsilon-\mathbf{D P}(X)$, then there exists $x \in X$ such that

$$
\left|\lambda_{\varphi}(\{x\})\right| \geq \frac{1+\sqrt{1-4 \epsilon}}{k} .
$$


Proof. By Lemma 3.2, we can assume, without loss of generality, that $\varphi$ is positive. Suppose that $k=2 m, m \in \mathbb{N}$. Notice that there cannot be $m$ different points $x_{1}, \ldots, x_{m} \in X$ with

$$
\lambda_{\varphi}\left(\left\{x_{i}\right\}\right) \in\left(\frac{1-\sqrt{1-4 \epsilon}}{k}, \frac{1+\sqrt{1-4 \epsilon}}{k}\right)
$$

for every $i \in\{1, \ldots, m\}$, because otherwise

$$
\lambda_{\varphi}\left(\left\{x_{1}, \ldots, x_{m}\right\}\right) \in\left(\frac{1-\sqrt{1-4 \epsilon}}{2}, \frac{1+\sqrt{1-4 \epsilon}}{2}\right),
$$

which contradicts Lemma 3.1. This implies that there exist at least $m+1$ points whose measure belongs to

$$
\left[0, \frac{1-\sqrt{1-4 \epsilon}}{k}\right] \cup\left[\frac{1+\sqrt{1-4 \epsilon}}{k}, 1\right] .
$$

Suppose that at least $m$ different points $x_{1}, \ldots, x_{m} \in X$ satisfy the inequality $\lambda_{\varphi}\left(\left\{x_{i}\right\}\right) \leq(1-\sqrt{1-4 \epsilon}) / k$. Then $\lambda_{\varphi}\left(\left\{x_{1}, \ldots, x_{m}\right\}\right) \leq(1-\sqrt{1-4 \epsilon}) / 2$, and consequently we have that $\lambda_{\varphi}\left(X \backslash\left\{x_{1}, \ldots, x_{m}\right\}\right) \geq(1+\sqrt{1-4 \epsilon}) / 2$. Since $X \backslash\left\{x_{1}, \ldots, x_{m}\right\}$ has $m$ points, this obviously implies that there exists $x \in X \backslash\left\{x_{1}, \ldots, x_{m}\right\}$ with $\lambda_{\varphi}(\{x\}) \geq(1+\sqrt{1-4 \epsilon}) / k$, and we are done.

Proposition 3.5. Let $0<\epsilon<1 / 4$, and let $n \in \mathbb{N}$ be such that $\epsilon \in \mathbb{A}_{n}$. Suppose that $\operatorname{card} X \geq 2 n$. If $\varphi \in \epsilon-\mathbf{D P}(X)$, then there exists $x \in X$ such that

$$
\left|\lambda_{\varphi}(\{x\})\right| \geq \frac{1+\sqrt{1-4 \epsilon}}{2 n} .
$$

Proof. Let $D:=\left\{x \in X:\left|\lambda_{\varphi}(\{x\})\right|>0\right\}$. It is clear that $D$ is a countable set, and, by Lemma 3.3. it is nonempty. Let $\mathbb{M}:=\{1, \ldots, m\}$ if the cardinality of $D$ is $m \in \mathbb{N}$, and let $\mathbb{M}:=\mathbb{N}$ otherwise. It is obvious that we may assume that $D=\left\{x_{i}: i \in \mathbb{M}\right\}$ and that $\left|\lambda_{\varphi}\left(\left\{x_{i+1}\right\}\right)\right| \leq\left|\lambda_{\varphi}\left(\left\{x_{i}\right\}\right)\right|$ for every $i$.

Next let

$$
\mathbb{J}:=\left\{j \in \mathbb{M}: \sum_{i=1}^{j}\left|\lambda_{\varphi}\left(\left\{x_{i}\right\}\right)\right|<\frac{1}{2}\right\}
$$

and

$$
R:=\sum_{i \in \mathbb{J}}\left|\lambda_{\varphi}\left(\left\{x_{i}\right\}\right)\right| .
$$

We have that $R \leq 1 / 2$, and by Lemma 3.1 applied to the functional associated to $\left|\lambda_{\varphi}\right|$, we get $R<1 / 2$. Take any open subset $U$ of $X$ containing all $x_{i}, i \in \mathbb{J}$, such that $\left|\lambda_{\varphi}\right|(U)<1 / 2$, that is, $\left|\lambda_{\varphi}\right|(U) \leq(1-\sqrt{1-4 \epsilon}) / 2$, and suppose that $\left|\lambda_{\varphi}(\{x\})\right|<\sqrt{1-4 \epsilon}$ for every $x \notin U$. Then there exist open sets $U_{1}, \ldots, U_{l}$ in $X$, $l \in \mathbb{N}$, such that $X=U \cup U_{1} \cup \cdots \cup U_{l}$ and $\left|\lambda_{\varphi}\right|\left(U_{i}\right)<\sqrt{1-4 \epsilon}$ for every $i$. If we consider, for $i \in\{1, \ldots, l\}, b_{i}:=\left|\lambda_{\varphi}\right|\left(U \cup \bigcup_{j=1}^{i} U_{j}\right)$, then we see that there must be an index $i_{0}$ with

$$
b_{i_{0}} \in\left(\frac{1-\sqrt{1-4 \epsilon}}{2}, \frac{1+\sqrt{1-4 \epsilon}}{2}\right),
$$

which contradicts Lemma 3.1 . 
We deduce that there exists $j \in \mathbb{M}, j \notin \mathbb{J}$, such that $\left|\lambda_{\varphi}\left(\left\{x_{j}\right\}\right)\right| \geq \sqrt{1-4 \epsilon}$. By the way we have taken $D$, this implies that $\left|\lambda_{\varphi}\left(\left\{x_{i}\right\}\right)\right| \geq \sqrt{1-4 \epsilon}$ for every $i \in \mathbb{J}$, and obviously $\mathbb{J}$ must be finite, say $\mathbb{J}=\left\{1, \ldots, m_{0}\right\}$.

Let us now see that $m_{0} \leq n-1$. We have that since $\epsilon<\omega_{2 n+1}$, then $\left.\sqrt{1-4 \epsilon}\right\rangle$ $1 /(2 n+1)$, which implies that

$$
n \sqrt{1-4 \epsilon}>\frac{1-\sqrt{1-4 \epsilon}}{2} .
$$

Consequently, if $m_{0} \geq n$, then we get

$$
\begin{aligned}
R & =\sum_{i=1}^{m_{0}}\left|\lambda_{\varphi}\left(\left\{x_{i}\right\}\right)\right| \\
& \geq n \sqrt{1-4 \epsilon} \\
& >\frac{1-\sqrt{1-4 \epsilon}}{2}
\end{aligned}
$$

which is impossible, as we said above. We conclude that $m_{0} \leq n-1$.

On the other hand, taking into account that

$$
\sum_{i=1}^{m_{0}+1}\left|\lambda_{\varphi}\left(\left\{x_{i}\right\}\right)\right| \geq \frac{1+\sqrt{1-4 \epsilon}}{2},
$$

we have that

$$
\left(m_{0}+1\right)\left|\lambda_{\varphi}\left(\left\{x_{1}\right\}\right)\right| \geq \frac{1+\sqrt{1-4 \epsilon}}{2}
$$

which implies that

$$
n\left|\lambda_{\varphi}\left(\left\{x_{1}\right\}\right)\right| \geq \frac{1+\sqrt{1-4 \epsilon}}{2} .
$$

As a consequence we get

$$
\left|\lambda_{\varphi}\left(\left\{x_{1}\right\}\right)\right| \geq \frac{1+\sqrt{1-4 \epsilon}}{2 n}
$$

and we are done.

Proof of Theorem 2.1. Let us show the first part. By Propositions 3.4 (see also the comments at the beginning of Section 3) and 3.5, there exists $x \in X$ with $\left|\lambda_{\varphi}(\{x\})\right| \geq 1-o_{X}(\epsilon)$. If we define $\psi:=\lambda_{\varphi}(\{x\}) \delta_{x}$, then we are done.

Let us now prove the second part. Suppose that $\epsilon$ belongs to $\mathbb{A}_{n}, n \in \mathbb{N}$. It is clear that this fact implies that $(2 n-1) \sqrt{1-4 \epsilon} \leq 1$.

If $\operatorname{card} X \geq 2 n$, then we can pick $2 n$ distinct points $x_{1}, x_{2}, \ldots, x_{2 n}$ in $X$ and define the map $\varphi \in C(X)^{\prime}$ as

$$
\varphi:=\frac{1+\sqrt{1-4 \epsilon}}{2 n}\left(\sum_{i=1}^{2 n-1} \delta_{x_{i}}\right)+\frac{1-(2 n-1) \sqrt{1-4 \epsilon}}{2 n} \delta_{x_{2 n}} .
$$

It is easy to see that $\|\varphi\|=1$. On the other hand, let $f, g \in C(X)$ satisfy $\|f\|_{\infty}=\|g\|_{\infty}=1$ and $f g \equiv 0$. Let $A_{f}:=\left\{x_{i}: f\left(x_{i}\right) \neq 0, i=1, \ldots, 2 n\right\}$ and $A_{g}:=\left\{x_{i}: g\left(x_{i}\right) \neq 0, i=1, \ldots, 2 n\right\}$, and suppose without loss of generality that $A_{f}=\left\{x_{1}, \ldots, x_{k}\right\}$ and $A_{g}=\left\{x_{k+1}, \ldots, x_{2 n}\right\}(1 \leq k \leq 2 n-1)$. Obviously

$$
|\varphi(f)| \leq \frac{k}{2 n}(1+\sqrt{1-4 \epsilon})
$$


and

$$
\begin{aligned}
|\varphi(g)| & \leq \frac{2 n-k-1}{2 n}(1+\sqrt{1-4 \epsilon})+\frac{1-(2 n-1) \sqrt{1-4 \epsilon}}{2 n} \\
& =1-\frac{k}{2 n}(1+\sqrt{1-4 \epsilon}) .
\end{aligned}
$$

Now, it is straightforward to check that if $N_{k}:=(k / 2 n)(1+\sqrt{1-4 \epsilon})$, then $N_{n-1} \leq 1-N_{n}$. Consequently, since the map $f(x):=x(1-x)$ is increasing in $[0,1 / 2]$, the maximum value of $N_{k}\left(1-N_{k}\right)$ is attained when $k=n$. Thus we conclude that

$$
|\varphi(f) \varphi(g)| \leq \frac{1}{2}(1+\sqrt{1-4 \epsilon})\left(1-\frac{1}{2}(1+\sqrt{1-4 \epsilon})\right)=\epsilon .
$$

The rest is easy.

To study the cases when $\operatorname{card} X<2 n$, put $X:=\left\{x_{1}, \ldots, x_{k}\right\}$. Suppose first that $k$ is even. Since $(2 n-1) \sqrt{1-4 \epsilon} \leq 1$, we have $(k-1) \sqrt{1-4 \epsilon}<1$. As above, we can easily see that if we define the $\operatorname{map} \varphi$ as

$$
\varphi:=\frac{1+\sqrt{1-4 \epsilon}}{k}\left(\sum_{i=1}^{k-1} \delta_{x_{i}}\right)+\frac{1-(k-1) \sqrt{1-4 \epsilon}}{k} \delta_{x_{k}},
$$

then we are done.

Suppose finally that $k$ is odd. It is clear that if we define

$$
\varphi:=\frac{1}{k}\left(\sum_{i=1}^{k} \delta_{x_{i}}\right)
$$

then $\varphi$ is a norm one element of $C(X)^{\prime}$ and is $\omega_{k}$-disjointness preserving, which implies that it is $\epsilon$-disjointness preserving. It is also easy to see that $\|\varphi-\psi\| \geq$ $1-1 / k$ for every weighted evaluation functional $\psi$ on $C(X)$.

Proof of Proposition 2.2. First, when card $X \geq 2 n$, we have that

$$
o_{X}(\epsilon)=\frac{2 n-1-\sqrt{1-4 \epsilon}}{2 n}
$$

for all $\epsilon \in \mathbb{A}_{n}$, and $\alpha_{2 n-1}:=o_{X}\left(\omega_{2 n-1}\right)$ and $\beta_{2 n}:=\lim _{\epsilon \rightarrow \omega_{2 n+1}^{-}} o_{X}(\epsilon)$, so $o_{X}\left(\mathbb{A}_{n}\right)=$ $\left[\alpha_{2 n-1}, \beta_{2 n}\right)$.

Also

and consequently

$$
n\left(1-o_{X}(\epsilon)\right)=\frac{1+\sqrt{1-4 \epsilon}}{2}
$$

$$
\epsilon=n\left(1-o_{X}(\epsilon)\right)\left(1-n\left(1-o_{X}(\epsilon)\right)\right),
$$

so $V_{X}\left(o_{X}(\epsilon)\right)=\epsilon$ for all $\epsilon \in \mathbb{A}_{n}$.

On the other hand, if card $X=2 m \in \mathbb{N}$, then

$$
o_{X}(\epsilon)=\frac{2 m-1-\sqrt{1-4 \epsilon}}{2 m}
$$

for every $\epsilon \in\left[\omega_{2 m+1}, 1 / 4\right)$. This means that $o_{X}\left[\omega_{2 m+1}, 1 / 4\right)=\left[\beta_{2 m}, \alpha_{2 m}\right)$ and that, for $\epsilon \in\left[\omega_{2 m+1}, 1 / 4\right)$,

$$
\epsilon=m\left(1-o_{X}(\epsilon)\right)\left(1-m\left(1-o_{X}(\epsilon)\right)\right)
$$

so the inverse of $o_{X}$ on $\left[\beta_{2 m}, \alpha_{2 m}\right)$ is also given by $V_{X}$.

All other details are straightforward. 


\section{ACKNOWLEDGEMENTS}

The authors wish to thank the referee for valuable remarks which improved this paper.

\section{REFERENCES}

[1] J. Araujo, E. Beckenstein and L. Narici, Biseparating maps and homeomorphic realcompactifications. J. Math. Anal. Appl. 192 (1995), 258-265. MR1329423 (96b:46038)

[2] J. Araujo and Juan J. Font, Stability of weighted composition operators between spaces of continuous functions. J. London Math. Soc (2) 79 (2009), 363-376. MR.2496519

[3] J. Araujo and Juan J. Font, On the stability index for weighted composition operators. Preprint.

[4] G. Dolinar, Stability of disjointness preserving mappings. Proc. Amer. Math. Soc. 130 (2002), 129-138. MR.1855629 (2002k:46126)

[5] J. J. Font and S. Hernández, On separating maps between locally compact spaces. Arch. Math. (Basel) 63 (1994), 158-165. MR.1289298 (95k:46083)

[6] K. Jarosz, Automatic continuity of separating linear isomorphisms. Canad. Math. Bull. 33 (1990), 139-144. MR.1060366 (92j:46049)

[7] J.-S. Jeang and N.-C. Wong, Weighted composition operators of $C_{0}(X)$ 's. J. Math. Anal. Appl. 201 (1996), 981-993. MR1400575 (97f:47029)

[8] B. E. Johnson, Approximately multiplicative functionals. J. London Math. Soc. (2) 34 (1986), 489-510. MR864452 (87k:46105)

[9] B. E. Johnson, Approximately multiplicative maps between Banach algebras. J. London Math. Soc. (2) 37 (1988), 294-316. MR928525 (89h:46072)

Departamento de Matemáticas, Estadística y Computación, Facultad de Ciencias, Universidad de Cantabria, Avda. De los Castros, S.n., E-39071 Santander, Spain

E-mail address: araujoj@unican.es

Departamento de Matemáticas, Universitat Jaume I, Campus Riu Sec, 8029 AP, Castellón, Spain

E-mail address: font@mat.uji.es 